ANNALES

POLONICI MATHEMATICI

$88.3(2006)$

\title{
Hölder continuity of weak solutions to nondiagonal singular parabolic systems of three equations
}

\author{
by Dmitry Portnyagin (Lviv)
}

\begin{abstract}
Hölder continuity of weak solutions is studied for a nondiagonal parabolic system of singular quasilinear differential equations with matrix of coefficients satisfying special structure conditions. A technique based on estimating linear combinations of the unknowns is employed.
\end{abstract}

1. Introduction. In the present paper we study the Hölder continuity of weak solutions to a quasilinear nondiagonal parabolic system of three singular equations in divergence form under special assumptions upon its structure.

It is well known that the De Giorgi-Nash-Moser estimates are no longer valid in general for elliptic systems; the latter can be regarded as a special case of parabolic systems. An example of an unbounded solution to a linear elliptic system with bounded coefficients was built up by De Giorgi in [11]. Another example is due to J. Nečas and J. Souček who constructed a nonlinear elliptic system with coefficients sufficiently smooth, but with a weak solution not belonging to $W^{2,2}$. These two and many other examples illustrate that the regularity problem for elliptic systems is far more complicated than for second order elliptic equations and that the smoothness properties of solutions are not only determined by the smoothness of data, but strongly depend upon the structure of the system.

Until now a priori estimates of De Giorgi type have been extended only to a special class of parabolic systems of equations, the so-called weakly coupled systems.

2000 Mathematics Subject Classification: Primary 35K50; Secondary 35D10, 35B65.

Key words and phrases: nondiagonal parabolic systems, Hölder continuity of solutions, singular equations.

This paper was written during the author's being a Ph.D. student at the Institute of Mathematics of the Polish Academy of Sciences, whose financial support is gratefully acknowledged. 
There exists yet another approach to establishing a priori estimates for a parabolic system of second order differential equations. This approach is used in the book by Ladyzhenskaya, Solonnikov and Ural'tseva [13], in the book by DiBenedetto [5] and in the papers [18] and [16]. It concerns not each component separately, but the sum of the squares of the components of a solution. This applies to diagonal systems which on freezing the leading coefficients and discarding the right-hand sides and lower order terms reduce to just one single equation rewritten several times in turn for all the unknown functions; see also [8], [10], [9], [6], [3, p. 27], [2, pp. 32-33] and [1].

The technique we are utilizing has been employed earlier in [14] for semilinear systems (see also [7], [15] and [12]), and consists in switching to new functions, for each of which the estimate is established in a conventional way, whence the final conclusion about each component of the vector function solution follows. This technique allows for extension to nondiagonal systems with nonlinearities in the spatial derivatives also.

The main idea of our approach is as follows: instead of trying to establish estimates for each component of a solution $(u, v, w)$ we introduce some linear combinations of the components of the solution:

$$
\begin{aligned}
& H_{1}=\alpha_{1} u+\beta_{1} v+w, \\
& H_{2}=\alpha_{2} u+\beta_{2} v+w, \\
& H_{3}=\alpha_{3} u+\beta_{3} v+w,
\end{aligned}
$$

or more generally some functions $H$ of $t, x, u, v, w$, for each of which the estimates hold and from whose estimates we shall be able to derive estimates for the components of $(u, v, w)$.

In the present paper, restricting ourselves to systems of second order equations in divergence form with a special structure, we demonstrate Hölder continuity of solutions to a quasilinear singular parabolic system of three equations in which coupling occurs in the leading derivatives and whose leading coefficients depend on $x, u, v, w$ and $u_{x}, v_{x}, w_{x}$.

2. Basic notations and hypotheses. We shall be concerned with a system of three equations of the form

$$
\left\{\begin{array}{c}
u_{t}-\frac{\partial}{\partial x_{i}}\left(A_{i}^{(1)}\left(x, u, v, w, u_{x}, v_{x}, w_{x}\right)\right)=B^{(1)}\left(x, u, v, w, u_{x}, v_{x}, w_{x}\right), \\
v_{t}-\frac{\partial}{\partial x_{i}}\left(A_{i}^{(2)}\left(x, u, v, w, u_{x}, v_{x}, w_{x}\right)\right)=B^{(2)}\left(x, u, v, w, u_{x}, v_{x}, w_{x}\right), \\
w_{t}-\frac{\partial}{\partial x_{i}}\left(A_{i}^{(3)}\left(x, u, v, w, u_{x}, v_{x}, w_{x}\right)\right)=B^{(3)}\left(x, u, v, w, u_{x}, v_{x}, w_{x}\right),
\end{array}\right.
$$


for $x \in Q=(0, T] \times \Omega$. Boundary conditions of the Dirichlet type are assumed:

$$
\left\{\begin{array}{l}
\left(u-g_{1}, v-g_{2}, w-g_{3}\right)(x, t) \in W_{0}^{1, p}(\Omega) \quad \text { a.e. } t \in(0, T), \\
(u, v, w)(x, 0)=\left(u_{0}, v_{0}, w_{0}\right)(x)
\end{array}\right.
$$

Solutions to system (2.1) with Dirichlet data (2.2) are defined as follows.

Definition 2.1. A measurable bounded vector function $\left(u^{1}, u^{2}, u^{3}\right)=$ $(u, v, w)$ is called a weak solution of problem $(2.1)-(2.2)$ if

$$
u^{j} \in C\left(0, T ; L^{2}(\Omega)\right) \cap L^{p}\left(0, T ; W^{1, p}(\Omega)\right) \cap L^{\infty}(Q)
$$

and for all $t \in(0, T]$,

$$
\begin{aligned}
\int_{\Omega} u^{j} \varphi_{j}(x, t) d x+\iint_{\Omega \times(0, t]}\left\{-u^{j} \varphi_{j t}\right. & \left.+A_{i}^{(j)} \varphi_{j} x_{i}\right\} d x d \tau \\
& =\int_{\Omega} u_{0}^{j} \varphi_{j}(x, 0) d x+\iint_{\Omega \times(0, t]} B^{j} \varphi_{j} d x d \tau
\end{aligned}
$$

for all bounded test functions

$$
\varphi \in W^{1,2}\left(0, T ; L^{2}(\Omega)\right) \cap L^{p}\left(0, T ; W_{0}^{1, p}(\Omega)\right), \quad \varphi \geq 0 .
$$

The boundary condition in (2.2) is meant in the weak sense.

Let us also define the boundary norms that will come in useful in further considerations.

Definition 2.2. Let $\Omega$ be a domain in $\mathbb{R}^{n}$ (here $n$ is any natural number) and $\partial \Omega$ a portion of its boundary; let $W(\Omega)$ be any Sobolev space. For a function $u$ defined on $\partial \Omega$ we set

$$
\|u\|_{W(\partial \Omega)}=\inf _{\psi}\|\psi\|_{W(\Omega)},
$$

where the infimum is taken over all functions $\psi \in W(\Omega)$ such that $\psi(x)=$ $u(x)$ a.e. on $\partial \Omega$. We denote by $W(\partial \Omega)$ the function space for which the aforementioned norm is finite.

Let us describe the notions, quantities and functions that will appear in this paper.

Here and onward we adopt the following notations: $Q=(0, T] \times \Omega$; $S=\partial \Omega \times(0, T] ; \partial Q \equiv\{\Omega \times\{0\}\} \cup\{\partial \Omega \times(0, T]\} ; \Omega$ is a bounded domain in $\mathbb{R}^{n}$ with piecewise smooth boundary; $x \in \Omega ; T>0 ; t \in(0, T] ; 1<p<2$; $p<n ; i=1, \ldots, n ; j=1,2,3$ and summation convention over repeated indices is assumed; $u, v, w \in C\left(0, T ; L^{2}(\Omega)\right) \cap L^{p}\left(0, T ; W^{1, p}(\Omega)\right) ; W_{0}^{1, p}(\Omega)$ is the space of functions in $W^{1, p}(\Omega)$ vanishing on $\partial \Omega$ in the sense of traces for a.e. $t \in(0, T]$. Throughout the paper, for brevity, $|s|$ and $\left|s_{i}\right|$ denote the 
distance in $3 n$-dimensional and $n$-dimensional Euclidean spaces respectively, i.e.

$$
|s|=\left(\sum_{j=1}^{3} \sum_{i=1}^{n}\left(s_{i}^{j}\right)^{2}\right)^{1 / 2}, \quad\left|s_{i}\right|=\left(\sum_{i=1}^{n}\left(s_{i}^{j}\right)^{2}\right)^{1 / 2}
$$

where $s_{i}^{j}$ stands for a $3 n$-component vector.

By parabolicity of system (2.1) it is meant that the part without time derivatives is elliptic. The notion of ellipticity of a system of differential equations is understood in the following sense (see [4]): there are $\lambda>0$ and $0<F=F(x) \in L^{p /(p-1)}(Q)$ such that for all $s_{i}^{j} \in \mathbb{R}^{3 n}, r_{(j)} \in \mathbb{R}^{3}$, and $x \in \mathbb{R}^{n}$,

$$
A_{i}^{(j)}(x, r, s) s_{i}^{j} \geq \lambda|s|^{p}-F .
$$

It should be emphasized that we impose neither the Legendre nor the Legendre-Hadamard condition. Both produce an obstacle from the technical point of view in the approach we take, while the ellipticity condition (2.3) turns out to be the most appropriate for our ends.

Moreover, it is assumed that $A_{i}^{(j)}: \Omega \times \mathbb{R}^{3} \times \mathbb{R}^{3 n} \rightarrow \mathbb{R}$ are measurable functions subject to the following growth condition: there exists $\Lambda_{2}>0$ such that for all $s_{i}^{j} \in \mathbb{R}^{3 n}, r^{j} \in \mathbb{R}^{3}$ and $x \in \mathbb{R}^{n}$,

$$
\left|A_{i}^{(j)}(x, r, s)\right| \leq \Lambda_{2}|s|^{p-1},
$$

and to the following structure conditions: there exist $\alpha_{j}, \beta_{j} \in \mathbb{R}$ with

$$
\operatorname{det}\left|\begin{array}{ccc}
\alpha_{1} & \alpha_{2} & \alpha_{3} \\
\beta_{1} & \beta_{2} & \beta_{3} \\
1 & 1 & 1
\end{array}\right| \neq 0
$$

such that for all $s_{i}^{j} \in \mathbb{R}^{3 n}, r^{j} \in \mathbb{R}^{3}$, and $x \in \mathbb{R}^{n}$,

$$
\begin{aligned}
\mid \alpha_{1} A_{i}^{(1)}(x, r, s) & +\beta_{1} A_{i}^{(2)}(x, r, s)+A_{i}^{(3)}(x, r, s) \\
& -\lambda_{1}(x, r, s)\left(\alpha_{1} s_{i}^{1}+\beta_{1} s_{i}^{2}+s_{i}^{3}\right) \mid \leq \xi_{1}(x, r, s)+F_{1} \\
\mid \alpha_{2} A_{i}^{(1)}(x, r, s) & +\beta_{2} A_{i}^{(2)}(x, r, s)+A_{i}^{(3)}(x, r, s) \\
& -\lambda_{2}(x, r, s)\left(\alpha_{2} s_{i}^{1}+\beta_{2} s_{i}^{2}+s_{i}^{3}\right) \mid \leq \xi_{2}(x, r, s)+F_{2}, \\
\mid \alpha_{3} A_{i}^{(1)}(x, r, s) & +\beta_{3} A_{i}^{(2)}(x, r, s)+A_{i}^{(3)}(x, r, s) \\
& -\lambda_{3}(x, r, s)\left(\alpha_{3} s_{i}^{1}+\beta_{3} s_{i}^{2}+s_{i}^{3}\right) \mid \leq \xi_{3}(x, r, s)+F_{3}
\end{aligned}
$$

here $\lambda_{j}=\lambda_{j}(x, r, s)>0$ and $\xi_{j}=\xi_{j}(x, r, s)>0$ are some measurable $\Omega \times \mathbb{R}^{3} \times \mathbb{R}^{3 n} \rightarrow \mathbb{R}$ functions of $x, u, v, w, u_{x}, v_{x}, w_{x}$ on which the following growth conditions are imposed: there exist $\Lambda_{1}, \Lambda_{2}>0$ such that for all 
$s_{i}^{j} \in \mathbb{R}^{3 n}, r^{j} \in \mathbb{R}^{3}$ and $x \in \mathbb{R}^{n}$,

$$
\begin{gathered}
0<\Lambda_{1}\left|\alpha_{j} s_{i}^{1}+\beta_{j} s_{i}^{2}+s_{i}^{3}\right|^{p-2} \leq \lambda_{j}(x, r, s) \leq \Lambda_{2}\left|\alpha_{j} s_{i}^{1}+\beta_{j} s_{i}^{2}+s_{i}^{3}\right|^{p-2} \\
\xi_{j}(x, r, s) \leq \xi_{0}|s|^{\nu}, \quad 0<\nu=\frac{p(p-1)\left(1-\kappa_{1}\right)}{(n+p)}
\end{gathered}
$$

where $\xi_{0}$ is a positive number;

$$
F_{j}(x, t) \in L^{\sigma}(Q), \quad \sigma=\frac{(p+n)}{(p-1)\left(1-\kappa_{1}\right)}, \quad \kappa_{1} \in(0,1),
$$

moreover

$$
\begin{gathered}
\alpha_{1}, \beta_{2}>1 ; \\
\alpha_{2}, \alpha_{3}, \beta_{1} \beta_{3}<1 ; \\
3 \max \left[1 / p, \Lambda_{2}\right] \max \left[\alpha_{1}^{-1}, \beta_{2}^{-1}, \alpha_{3}, \beta_{3}\right] \leq \Lambda_{1} /\left(2^{p} p\right) ; \\
6 \xi_{0} \leq \Lambda_{1} /\left(2^{p+1} p\right) .
\end{gathered}
$$

REMARK 1. It is not difficult to check by direct calculation, taking into account the fact that $F_{j} \in L^{(p+n) /\left((p-1)\left(1-\kappa_{1}\right)\right)}$, that the structure conditions (2.5a)-(2.5c) along with (2.6) and (2.12) imply the ellipticity condition (2.3) with $\lambda=\Lambda_{1} /\left(2^{p+1} p\right)$ and $F \equiv C_{1}\left(\left|F_{1}\right|+\left|F_{2}\right|+\left|F_{3}\right|\right)^{p /(p-1)}+C_{2}$, where $C_{1,2}$ are numbers depending only on the data.

REMARK 2. When $\alpha_{1}, \beta_{2} \rightarrow \infty$ and $\alpha_{2}, \alpha_{3}, \beta_{1}, \beta_{3}=0$ our system turns into a diagonal one with a slight perturbation. Earlier such systems (called weakly nondiagonal systems) were studied in [19], [17], [20] for the degenerate case.

The right-hand sides $B^{j}(x, r, s)$ are assumed to be measurable $\Omega \times \mathbb{R}^{3} \times$ $\mathbb{R}^{3 n} \rightarrow \mathbb{R}$ functions satisfying: there exist

$$
\varepsilon \in\left(0, \min \left\{\frac{p^{2}\left(1-\kappa_{1}\right)}{(n+p)}, p-1\right\}\right]
$$

and $\Lambda_{3}>0$ such that for all $s_{i}^{j} \in \mathbb{R}^{3 n}, r^{j} \in \mathbb{R}^{3}$ and $x \in \mathbb{R}^{n}$,

$$
\left|B^{j}(x, r, s)\right| \leq \Lambda_{3}|s|^{\varepsilon} \text {. }
$$

In what follows for brevity we use the notations:

$$
\begin{aligned}
& \widetilde{u}_{0}= \begin{cases}u_{0}(x), & x \in \Omega, t=0, \\
g_{1}(x, t), & x \in \partial \Omega, t \in(0, T) ;\end{cases} \\
& \widetilde{v}_{0}= \begin{cases}v_{0}(x), & x \in \Omega, t=0, \\
g_{2}(x, t), & x \in \partial \Omega, t \in(0, T) ;\end{cases} \\
& \widetilde{w}_{0}= \begin{cases}w_{0}(x), & x \in \Omega, t=0, \\
g_{3}(x, t), & x \in \partial \Omega, t \in(0, T) .\end{cases}
\end{aligned}
$$


Let us introduce in addition the following function space:

DEFINITION 2.3.

$$
\widetilde{W}(Q)=L^{p^{\prime}}\left(W^{1, p^{\prime}}(0, T) ; \Omega\right) \cap L^{p}\left(0, T ; W^{1, p}(\Omega)\right), \quad p^{\prime}=\frac{p}{p-1} ;
$$

i.e. a function $u$ belongs to $\widetilde{W}(Q)$ if the integral

$$
\int_{0}^{T} \int_{\Omega}\left(\left|u_{t}\right|^{p^{\prime}}+|\nabla u|^{p}+|u|^{p}+|u|^{p^{\prime}}\right)
$$

is finite.

The functions $g_{j}(x, t),\left(u_{0}, v_{0}, w_{0}\right)(x)$ in boundary data $(2.2)$ are assumed to satisfy

$$
\widetilde{u}_{0} \in \widetilde{W}(\partial Q), \quad \widetilde{v}_{0} \in \widetilde{W}(\partial Q), \quad \widetilde{w}_{0} \in \widetilde{W}(\partial Q) ;
$$

and, in addition, for some $\alpha_{g} \in(0,1)$ and $\alpha_{0} \in(0,1)$ their values on $\partial Q$ satisfy

$$
g_{j}(x, t) \in H^{\alpha_{g}, \alpha_{g} / p}(S), \quad\left(u_{0}, v_{0}, w_{0}\right)(x) \in H^{\alpha_{0}}(\bar{\Omega} \times\{0\}),
$$

where $H^{\alpha_{g}, \alpha_{g} / p}$ and $H^{\alpha_{0}}$ denote Hölder spaces with exponents $\alpha_{g}$ and $\alpha_{0}$ respectively.

3. Estimate for the sum of squares. We need to estimate the integral of the sum of the squares of the spatial derivatives of the components of a solution of problem (2.1)-(2.2).

Our goal in this section is to prove the following statement.

THEOREM 3.1. Let $(u, v, w)$ be a solution to problem (2.1)-(2.2) and suppose the hypotheses (2.5a)-(2.5c), (2.6), (2.7)-(2.12) and (2.13) are satisfied. Then

$$
\begin{aligned}
\sup _{0<t<T} \int_{\Omega}\left|u-\widetilde{u}_{0}\right|^{2}+\sup _{0<t<T} \int_{\Omega}\left|v-\widetilde{v}_{0}\right|^{2}+\sup _{0<t<T} \int_{\Omega}\left|w-\widetilde{w}_{0}\right|^{2} \\
+\int_{0}^{T} \int_{\Omega}\left(\left|\nabla\left(u-\widetilde{u}_{0}\right)\right|^{p}+\left|\nabla\left(v-\widetilde{v}_{0}\right)\right|^{p}+\left|\nabla\left(w-\widetilde{w}_{0}\right)\right|^{p}\right) \leq C
\end{aligned}
$$

and

$$
\int_{0}^{T} \int_{\Omega}\left(|\nabla u|^{p}+|\nabla v|^{p}+|\nabla w|^{p}\right) \leq C
$$

with a constant $C$ depending only on the data: $F_{j},\left\|\widetilde{u}_{0}\right\|_{\widetilde{W}(\partial Q)},\left\|\widetilde{v}_{0}\right\|_{\widetilde{W}(\partial Q)}$, $\left\|\widetilde{w}_{0}\right\|_{\widetilde{W}(\partial Q)}, p, n, \Lambda_{1}, \Lambda_{2}, \xi_{0}, \kappa_{1}, \alpha_{j}, \beta_{j}, \varepsilon$, mes $Q$, and independent of $u, v$ and $w$. 
REMARK 3 . In the formulation of the theorem and in its proof, by $\widetilde{u}_{0}, \widetilde{v}_{0}$ and $\widetilde{w}_{0}$ are meant any functions from $\widetilde{W}(Q)$ coinciding with $\widetilde{u}_{0}, \widetilde{v}_{0}$ and $\widetilde{w}_{0}$ on the parabolic boundary. Therefore the final statement remains valid with the boundary norms.

Proof. Multiply the first equation of (2.1) by $u-\widetilde{u}_{0}$, the second by $v-\widetilde{v}_{0}$, and the third by $w-\widetilde{w}_{0}$. After adding the results and integrating over $\Omega \times$ $(0, t)$ we obtain

$$
\begin{aligned}
\int_{\Omega(t)} \frac{1}{2}(u & \left.-\widetilde{u}_{0}\right)^{2}+\int_{\Omega(t)} \frac{1}{2}\left(v-\widetilde{v}_{0}\right)^{2}+\int_{\Omega(t)} \frac{1}{2}\left(w-\widetilde{w}_{0}\right)^{2} \\
& +\int_{0}^{t} \int_{\Omega} \vec{A}^{(1)} \nabla\left(u-\widetilde{u}_{0}\right)+\int_{0}^{t} \int_{\Omega} \vec{A}^{(2)} \nabla\left(v-\widetilde{v}_{0}\right)+\int_{0}^{t} \int_{\Omega} \vec{A}^{(3)} \nabla\left(w-\widetilde{w}_{0}\right) \\
\leq & \int_{0}^{t} \int_{\Omega}^{t}\left|B^{(1)}\right|\left|u-\widetilde{u}_{0}\right|+\int_{0}^{t} \int_{\Omega}\left|B^{(2)}\right|\left|v-\widetilde{v}_{0}\right|+\int_{0}^{t} \int_{\Omega}\left|B^{(3)}\right|\left|w-\widetilde{w}_{0}\right| \\
& +\int_{0}^{t} \int_{\Omega}\left|\widetilde{u}_{0 t}\right|\left|u-\widetilde{u}_{0}\right|+\int_{0}^{t} \int_{\Omega}\left|\widetilde{v}_{0 t}\right|\left|v-\widetilde{v}_{0}\right|+\int_{0}^{t}\left|\widetilde{w}_{0 t}\right|\left|w-\widetilde{w}_{0}\right|,
\end{aligned}
$$

where integration by parts with respect to the time variable was performed in the first two terms and the initial condition was taken into account. By the ellipticity condition (2.3) and growth conditions (2.4), the second group of terms on the left admits an estimate

$$
\begin{aligned}
& \int_{0}^{t} \int_{\Omega}\left(\vec{A}^{(1)} \nabla\left(u-\widetilde{u}_{0}\right)+\vec{A}^{(2)} \nabla\left(v-\widetilde{v}_{0}\right)+\vec{A}^{(3)} \nabla\left(w-\widetilde{w}_{0}\right)\right) \\
&= \int_{0}^{t} \int_{\Omega}\left(\vec{A}^{(1)} \nabla u+\vec{A}^{(2)} \nabla v+\vec{A}^{(3)} \nabla w-\vec{A}^{(1)} \nabla \widetilde{u}_{0}-\vec{A}^{(2)} \nabla \widetilde{v}_{0}-\vec{A}^{(3)} \nabla \widetilde{w}_{0}\right) \\
& \geq \int_{0}^{t} \int_{\Omega} \lambda\left(|\nabla u|^{p}+|\nabla v|^{p}+|\nabla w|^{p}\right)-\int_{0}^{t} \int_{\Omega} \lambda\left(|\nabla u|^{p-1}+|\nabla v|^{p-1}+|\nabla w|^{p-1}\right) \\
& \times\left(\left|\nabla u_{0}\right|+\left|\nabla v_{0}\right|+\left|\nabla w_{0}\right|\right)-\int_{0}^{t} \int_{\Omega}\left(\left|F_{1}\right|+\left|F_{2}\right|+\left|F_{3}\right|\right) \\
& \geq \int_{0}^{t} \int_{\Omega} \frac{1}{2} \lambda\left(|\nabla u|^{p}+|\nabla v|^{p}+|\nabla w|^{p}\right) \\
&-C(p, \lambda) \int_{0}^{t} \int_{\Omega}\left(\left|\nabla u_{0}\right|^{p}+\left|\nabla v_{0}\right|^{p}+\left|\nabla w_{0}\right|^{p}\right)-C
\end{aligned}
$$




$$
\begin{aligned}
& \geq \int_{0}^{t} \int_{\Omega} \frac{1}{2} \lambda\left(\left|\nabla\left(u-\widetilde{u}_{0}\right)\right|^{p}+\left|\nabla\left(v-\widetilde{v}_{0}\right)\right|^{p}+\left|\nabla\left(w-\widetilde{w}_{0}\right)\right|^{p}\right) \\
& \quad-\int_{0}^{t} \int_{\Omega} \widetilde{C}(p, \lambda)\left(\left|\nabla u_{0}\right|^{p}+\left|\nabla v_{0}\right|^{p}+\left|\nabla w_{0}\right|^{p}\right)-C .
\end{aligned}
$$

Here use is also made of Young's inequality and the inequality

$$
|a+b|^{p} \leq C(p)\left(|a|^{p}+|b|^{p}\right), \quad \forall a, b \in \mathbb{R} .
$$

In view of Young's inequality, the Sobolev inequality and growth condition (2.13) the first three terms on the right of (3.1) can be estimated as follows:

$$
\begin{aligned}
& \int_{0}^{t} \int_{\Omega}\left|B^{(1)}\right|\left|u-\widetilde{u}_{0}\right|+\int_{0} \int_{\Omega}\left|B^{(2)}\right|\left|v-\widetilde{v}_{0}\right|+\int_{0}^{t}\left|B_{\Omega}^{(3)}\right|\left|w-\widetilde{w}_{0}\right| \\
& \leq \int_{0}^{t} \int_{\Omega}(|\nabla u|+|\nabla v|+|\nabla w|)^{\varepsilon}\left(\left|u-\widetilde{u}_{0}\right|+\left|v-\widetilde{v}_{0}\right|+\left|w-\widetilde{w}_{0}\right|\right) \\
& \leq \delta_{1} C_{1}(\varepsilon, p) \int_{0}^{t} \int_{\Omega}\left(\left|\nabla\left(u-\widetilde{u}_{0}\right)\right|+\left|\nabla\left(v-\widetilde{v}_{0}\right)\right|+\left|\nabla\left(w-\widetilde{w}_{0}\right)\right|\right)^{p} \\
& \quad+\delta_{2} C_{2}(p) \int_{0}^{t}\left(\left|u-\widetilde{u}_{0}\right|+\left|v-\widetilde{v}_{0}\right|+\left|w-\widetilde{w}_{0}\right|\right)^{p} \\
& \quad+C\left(C_{1,2}, \delta_{1,2}, \widetilde{u}_{0}, \widetilde{v}_{0}, \widetilde{w}_{0}, \operatorname{mes} Q\right) \\
& \quad \delta_{3} \int_{0}^{t}\left(\left|\nabla\left(u-\widetilde{u}_{0}\right)\right|+\left|\nabla\left(v-\widetilde{v}_{0}\right)\right|+\left|\nabla\left(w-\widetilde{w}_{0}\right)\right|\right)^{p}+C_{3} .
\end{aligned}
$$

Here it has been taken into account that $\varepsilon / p+1 / p<1$. In much the same way we can estimate the last three integrals on the right-hand side of (3.1):

$$
\begin{aligned}
& \int_{0}^{t} \int_{\Omega}\left|\widetilde{u}_{0 t}\right|\left|u-\widetilde{u}_{0}\right|+\int_{0}^{t} \int_{\Omega}\left|\widetilde{v}_{0 t}\right|\left|v-\widetilde{v}_{0}\right|+\int_{0}^{t}\left|\widetilde{w}_{0 t}\right|\left|w-\widetilde{w}_{0}\right| \\
& \leq \int_{0}^{t} \int_{\Omega}\left(\left|\widetilde{u}_{0 t}\right|+\left|\widetilde{v}_{0 t}\right|+\left|\widetilde{w}_{0 t}\right|\right)\left(\left|u-\widetilde{u}_{0}\right|+\left|v-\widetilde{v}_{0}\right|+\left|w-\widetilde{w}_{0}\right|\right) \\
& \leq \|\left|\widetilde{u}_{0 t}\right|+\left|\widetilde{v}_{0 t}\right|+\left|\widetilde{w}_{0 t}\right|||_{p^{\prime}, Q}\left(\int_{0}^{t} \int_{\Omega}\left(\left|u-\widetilde{u}_{0}\right|+\left|v-\widetilde{v}_{0}\right|+\left|w-\widetilde{w}_{0}\right|\right)^{p}\right)^{1 / p} \\
& \leq \delta_{4} \int_{0}^{t}\left(\left|\nabla\left(u-\widetilde{u}_{0}\right)\right|+\left|\nabla\left(v-\widetilde{v}_{0}\right)\right|+\left|\nabla\left(w-\widetilde{w}_{0}\right)\right|\right)^{p} \\
& \quad+C_{4}\left(\operatorname{mes} Q, \delta_{4}, \widetilde{u}_{0}, \widetilde{v}_{0}, \widetilde{w}_{0}\right) .
\end{aligned}
$$


Collecting the above estimates, from (3.1) we get

$$
\begin{aligned}
& \int_{\Omega(t)} \frac{1}{2}\left[\left(u-\widetilde{u}_{0}\right)^{2}+\right. \\
& \left.\left.+v-\widetilde{v}_{0}\right)^{2}+\left(w-\widetilde{w}_{0}\right)^{2}\right] \\
& \quad+\int_{0}^{t} \frac{1}{2} \lambda\left(\left|\nabla\left(u-\widetilde{u}_{0}\right)\right|^{p}+\left|\nabla\left(v-\widetilde{v}_{0}\right)\right|^{p}+\left|\nabla\left(w-\widetilde{w}_{0}\right)\right|^{p}\right) \\
& \leq \delta_{5} \int_{0}^{t} \int_{\Omega}\left(\left|\nabla\left(u-\widetilde{u}_{0}\right)\right|^{p}+\left|\nabla\left(v-\widetilde{v}_{0}\right)\right|^{p}+\left|\nabla\left(w-\widetilde{w}_{0}\right)\right|^{p}\right) \\
& \quad+C_{5}\left(\operatorname{mes} Q, F, \delta_{5}, \widetilde{u}_{0}, \widetilde{v}_{0}, \widetilde{w}_{0}\right) .
\end{aligned}
$$

Choosing $\delta_{5}=\frac{1}{4} \lambda$ yields

$$
\begin{aligned}
& \int_{\Omega(t)} \frac{1}{2}\left[\left(u-\widetilde{u}_{0}\right)^{2}+\left(v-\widetilde{v}_{0}\right)^{2}+\left(w-\widetilde{w}_{0}\right)^{2}\right] \\
& +\int_{0}^{t} \int_{\Omega} \frac{1}{4} \lambda\left(\left|\nabla\left(u-\widetilde{u}_{0}\right)\right|^{p}+\left|\nabla\left(v-\widetilde{v}_{0}\right)\right|^{p}+\left|\nabla\left(w-\widetilde{w}_{0}\right)\right|^{p}\right) \\
& \leq C_{4}\left(\operatorname{mes} Q, F, \delta_{5}, \widetilde{u}_{0}, \widetilde{v}_{0}, \widetilde{w}_{0}\right) .
\end{aligned}
$$

Now we take the supremum over $t$ on the left-hand side of (3.3) to obtain

$$
\begin{aligned}
\sup _{0<t<T} \int_{\Omega}\left|u-\widetilde{u}_{0}\right|^{2}+\sup _{0<t<T} \int_{\Omega}\left|v-\widetilde{v}_{0}\right|^{2}+\sup _{0<t<T} \int_{\Omega}\left|w-\widetilde{w}_{0}\right|^{2} \\
+\int_{0}^{T} \int_{\Omega}\left(\left|\nabla\left(u-\widetilde{u}_{0}\right)\right|^{p}+\left|\nabla\left(v-\widetilde{v}_{0}\right)\right|^{p}+\left|\nabla\left(w-\widetilde{w}_{0}\right)\right|^{p}\right) \leq C_{5}
\end{aligned}
$$

with a constant $C_{5}$ depending on $n, p, \varepsilon, \lambda, F_{j}, p, n, \Lambda_{1}, \Lambda_{2}, \xi_{0}, \kappa_{1}, \alpha_{j}, \beta_{j}$, $\varepsilon$, mes $Q$ and, by Remark 3 , on the boundary norms $\left\|\widetilde{u}_{0}\right\|_{\widetilde{W}(\partial Q)},\left\|\widetilde{v}_{0}\right\|_{\widetilde{W}(\partial Q)}$ and $\left\|\widetilde{w}_{0}\right\|_{\widetilde{W}(\partial Q)}$ of the functions in the boundary conditions only. Hence the second statement of the theorem is self-evident.

\section{Hölder continuity of weak solutions}

Interior regularity. We introduce the number $\widehat{q} \geq 1$ such that

$$
\widehat{q}=\frac{(p+n)}{p\left(1-\kappa_{1}\right)}, \quad \kappa_{1} \in(0,1),
$$

and the numbers $q$ and $\kappa$ satisfying

$$
q=\frac{\widehat{q} p(1+\kappa)}{\widehat{q}-1}, \quad \kappa=\frac{p \kappa_{1}}{n} .
$$


Let $K_{\varrho}$ be the $n$-dimensional cube centered at the origin with edge $2 \varrho$ :

$$
K_{\varrho} \equiv\left\{x \in \mathbb{R}^{n}\left|\max _{i}\right| x_{i} \mid<\varrho\right\},
$$

and let $\left[x_{0}+K_{\varrho}\right]$ be its translate,

$$
\left[x_{0}+K_{\varrho}\right] \equiv\left\{x \in \mathbb{R}^{n}\left|\max _{i}\right| x_{i}-x_{0 i} \mid<\varrho\right\} ;
$$

let $Q(\theta, \varrho)$ be a cylinder of height $\theta$ built over the cube $K_{\varrho}$ :

$$
Q(\theta, \varrho) \equiv K_{\varrho} \times\{-\theta, 0\},
$$

and $\left[\left(x_{0}, t_{0}\right)+Q(\theta, \varrho)\right]$ be its translate,

$$
\left[\left(x_{0}, t_{0}\right)+Q(\theta, \varrho)\right] \equiv\left[x_{0}+K_{\varrho}\right] \times\left\{t_{0}-\theta, t_{0}\right\} ;
$$

let

$$
A_{k, \varrho}^{ \pm} \equiv\left\{x \in\left[x_{0}+K_{\varrho}\right] \mid(H(x, \tau)-k)_{ \pm}>0\right\},
$$

where $\varrho$ and $\theta$ are positive numbers so small that $\left[\left(x_{0}, t_{0}\right)+Q(\theta, \varrho)\right] \subset Q$; set

$$
M_{k}^{ \pm} \equiv \operatorname{essip}_{\left[\left(x_{0}, t_{0}\right)+Q(\theta, \varrho)\right]}\left|(H-k)_{ \pm}\right| \leq \delta \leq \delta_{0},
$$

where $\delta_{0}=\Lambda_{1} / 4 \Lambda_{2}$ is a positive parameter, and $\Lambda_{1}$ and $\Lambda_{2}$ are from (2.6); let $\zeta(x, t)$ be a piecewise smooth cutoff function in $\left[\left(x_{0}, t_{0}\right)+Q(\theta, \varrho)\right]$ with

$$
\begin{aligned}
& \zeta(x, t) \in[0,1], \quad|D \zeta|<\infty \quad \text { for } x \in\left[x_{0}+K_{\varrho}\right], \\
& \zeta(x, t) \equiv 0 \quad \text { for } x \notin\left[x_{0}+K_{\varrho}\right] .
\end{aligned}
$$

For every $\varphi \in L^{1}(Q)$ and $0<h<T$ we introduce the Steklov averages:

$$
\varphi_{h}(x, t) \equiv \begin{cases}\frac{1}{h} \int_{t}^{t+h} \varphi(x, \tau) d \tau, & t \in(0, T-h], \\ 0, & t>T-h,\end{cases}
$$

for all $0<t<T$. Recall that for $\varphi \in L^{q}(\Omega \times(0, T))$ we have $\varphi_{h} \rightarrow \varphi$ as $h \rightarrow 0$ in $L^{q}(\Omega \times(0, T-\varepsilon))$ for every $\varepsilon \in(0, T)$; and for $\varphi \in C\left(0, T ; L^{q}(\Omega)\right)$, $\varphi_{h}(t) \rightarrow \varphi(t)$ as $h \rightarrow 0$ in $L^{q}(\Omega)$ for every $t \in(0, T-\varepsilon), \forall \varepsilon \in(0, T)$.

According to the methodology set forth in [5], for Hölder continuity of weak solutions (Theorems 4.3 and 4.6 below) it is necessary to show the following propositions, Theorems 4.1-4.5:

TheOREM 4.1 (Local energy estimates). Let $(u, v, w)$ be a bounded solution to the system and $H=H_{j}$ be as in (1.1). There exist constants $\widetilde{C}$ and $\delta_{0}$ that can be determined a priori only in terms of the data such that for every cylinder $\left[\left(x_{0}, t_{0}\right)+Q(\theta, \varrho)\right] \subset Q$ and for every level $k$ satisfying (4.2) 
we have the estimate

$$
\begin{aligned}
\sup _{t_{0}-\theta<t<t_{0}} \int_{\left[x_{0}+K_{\varrho}\right]}(H-k)_{ \pm}^{2} \zeta^{p}(x, t) d x & \\
& +\widetilde{C}^{-1} \iiint_{\left[\left(x_{0}, t_{0}\right)+Q(\theta, \varrho)\right]}\left|\nabla(H-k)_{ \pm} \zeta\right|^{p} d x d \tau
\end{aligned}
$$

$$
\begin{aligned}
& \leq \int_{\left[x_{0}+K_{\varrho}\right]}(H-k)_{ \pm}^{2} \zeta^{p}\left(x, t_{0}-\theta\right) d x+\widetilde{C} \iiint_{\left[\left(x_{0}, t_{0}\right)+Q(\theta, \varrho)\right]}(H-k)_{ \pm}^{p}|\nabla \zeta|^{p} d x d \tau \\
& \quad+\widetilde{C} \iint_{\left[\left(x_{0}, t_{0}\right)+Q(\theta, \varrho)\right]}(H-k)_{ \pm}^{2} \zeta^{p-1} \zeta_{t} d x d \tau+\widetilde{C}\left\{\int_{t_{0}-\theta}^{t_{0}}\left|A_{k, \varrho}^{ \pm}(\tau)\right| d \tau\right\}^{p(1+\kappa) / q} .
\end{aligned}
$$

Proof. It suffices to prove (4.4) for the cube $Q(\theta, \varrho)$ since without loss of generality $\left(x_{0}, t_{0}\right)$ may be assumed to be the origin. Acting as in the previous section, multiply the first equation of system (2.1) by $\alpha\left(\alpha=\alpha_{1}, \alpha_{2}, \alpha_{3}\right)$, the second by $\beta$ ( $\left.\beta=\beta_{1}, \beta_{2}, \beta_{3}\right)$, then add the results and choose as test functions

$$
\varphi= \pm\left(H_{h}-k\right)_{ \pm} \zeta^{p}
$$

with $\zeta(x, t)$ satisfying (4.3). After integrating in $\tau$ over $(-\theta, t)$ with $t \in$ $(-\theta, 0)$, letting $h$ tend to zero, and making use of the structure conditions $(2.5 \mathrm{a})-(2.5 \mathrm{c})$ for the leading terms we get

$$
\begin{aligned}
& \quad \iint_{K_{\varrho} \times(-\theta, t)}\left\langle\alpha A^{(1)}+\beta A^{(2)}+A^{(3)}, \pm \nabla(H-k)_{ \pm} \zeta^{p} \pm p(H-k)_{ \pm} \zeta^{p-1} \nabla \zeta\right\rangle d x d \tau \\
& \geq \Lambda_{1} \iint_{K_{\varrho} \times(-\theta, t)}|\nabla H|^{p} \zeta^{p} d x d \tau
\end{aligned}
$$$$
-\Lambda_{2} \iint_{K_{\varrho} \times(-\theta, t)}|\nabla u, \nabla v, \nabla w|^{p(p-1)\left(1-\kappa_{1}\right) /(n+p)}|\nabla H| \zeta^{p} d x d \tau
$$$$
-\quad \iint_{K_{\varrho} \times(-\theta, t)} F|\nabla H| \zeta^{p} d x d \tau
$$$$
-p \Lambda_{2} \iint_{K_{\varrho} \times(-\theta, t)}|\nabla H|^{p-1}(H-k)_{ \pm} \zeta^{p-1}|\nabla \zeta| d x d \tau
$$

$-p \Lambda_{2} \iint_{K_{\varrho} \times(-\theta, t)}|\nabla u, \nabla v, \nabla w|^{p(p-1)\left(1-\kappa_{1}\right) /(n+p)}(H-k)_{ \pm} \zeta^{p-1}|\nabla \zeta| d x d \tau$

$$
-p \quad \iint_{K_{\varrho} \times(-\theta, t)} F(H-k)_{ \pm} \zeta^{p-1}|\nabla \zeta| d x d \tau
$$


Here and onward for brevity we write $|\nabla u, \nabla v, \nabla w|=\left(|\nabla u|^{2}+|\nabla v|^{2}+\right.$ $\left.|\nabla w|^{2}\right)^{1 / 2}$. Let us estimate various terms of the latter expression by Young's inequality.

For the first group of terms in (4.5), we get

$$
\begin{aligned}
& \quad \iint_{K_{\varrho} \times(-\theta, t)}|\nabla u, \nabla v, \nabla w|^{p(p-1)\left(1-\kappa_{1}\right) /(n+p)}|\nabla H| \zeta^{p} d x d \tau \\
& \leq C_{2}\left(p, \Lambda_{1}, \Lambda_{2}\right) \quad \iint_{K_{\varrho} \times(-\theta, t)}|\nabla u, \nabla v, \nabla w|^{p^{2}\left(1-\kappa_{1}\right) /(n+p)} \chi\left[(H-k)_{ \pm}>0\right] d x d \tau \\
& +\left(\Lambda_{1} / 6 \Lambda_{2}\right) \quad \iint_{K_{\varrho} \times(-\theta, t)}|\nabla H|^{p} \zeta^{p} d x d \tau ;
\end{aligned}
$$

$$
\begin{aligned}
& \iint_{K_{\varrho} \times(-\theta, t)} F|\nabla H| \zeta^{p} d x d \tau \\
& \leq C_{3}\left(p, \Lambda_{1}, \Lambda_{2}\right) \quad \iint_{K_{\varrho} \times(-\theta, t)} F^{p /(p-1)} \chi\left[(H-k)_{ \pm}>0\right] d x d \tau \\
& \quad+\left(\Lambda_{1} / 6\right) \iiint_{K_{\varrho} \times(-\theta, t)}|\nabla H|^{p} \zeta^{p} d x d \tau .
\end{aligned}
$$

For the second group of terms in (4.5) we obtain the following estimates:

$$
\begin{aligned}
& p \iint_{K_{\varrho} \times(-\theta, t)}|\nabla H|^{p-1}(H-k)_{ \pm} \zeta^{p-1}|\nabla \zeta| d x d \tau \\
& \leq C_{4}\left(p, \Lambda_{1}, \Lambda_{2}\right) \quad \iint_{K_{\varrho} \times(-\theta, t)}(H-k)_{ \pm}^{p}|\nabla \zeta|^{p} d x d \tau \\
& \quad+\left(\Lambda_{1} / 6 \Lambda_{2}\right) \quad \iint_{K_{\varrho} \times(-\theta, t)}|\nabla H|^{p} \zeta^{p} d x d \tau
\end{aligned}
$$

(4.9) $\quad p \quad \iint_{K_{0}}|\nabla u, \nabla v, \nabla w|^{p(p-1)\left(1-\kappa_{1}\right) /(n+p)}(H-k)_{ \pm} \zeta^{p-1}|\nabla \zeta| d x d \tau$ $K_{\varrho} \times(-\theta, t)$

$\leq(p-1) \quad \iint_{K_{\varrho} \times(-\theta, t)}|\nabla u, \nabla v, \nabla w|^{p^{2}\left(1-\kappa_{1}\right) /(n+p)} \chi\left[(H-k)_{ \pm}>0\right] d x d \tau$

$$
+\quad \iint_{K_{\varrho} \times(-\theta, t)}(H-k)_{ \pm}^{p}|\nabla \zeta|^{p} d x d \tau
$$

(4.10) $\quad p \quad \iint_{K_{\varrho} \times(-\theta, t)} F(H-k)_{ \pm} \zeta^{p-1}|\nabla \zeta| d x d \tau$

$\leq \int_{K_{\varrho} \times(-\theta, t)}(H-k)_{ \pm}^{p}|\nabla \zeta|^{p} d x d \tau+(p-1) \iint_{K_{\varrho} \times(-\theta, t)} F^{p /(p-1)} \chi\left[(H-k)_{ \pm}>0\right] d x d \tau$. 
Now we turn our attention to the right-hand sides of the system, i.e. to the terms containing $B$ 's. Making use of the growth assumptions (2.13) yields the following terms in the integral inequality:

$$
\begin{aligned}
& \pm \iint_{K_{\varrho} \times(-\theta, t)}\left(\alpha B^{(1)}+\beta B^{(2)}+B^{(3)}\right)(H-k)_{ \pm} \zeta^{p} d x d \tau \\
& \quad \leq \Lambda_{2}(|\alpha|+|\beta|+1) \quad \iint_{K_{\varrho} \times(-\theta, t)}|\nabla u, \nabla v, \nabla w|^{\varepsilon}(H-k)_{ \pm} \zeta^{p} d x d \tau .
\end{aligned}
$$

By the restriction on the set of levels in (4.2) we get

$$
\begin{aligned}
& \quad \pm \quad \iint_{K_{\varrho} \times(-\theta, t)}\left(\alpha B^{(1)}+\beta B^{(2)}+B^{(3)}\right)(H-k)_{ \pm} \zeta^{p} d x d \tau \\
& \leq C_{7}\left(p, \alpha, \beta, \Lambda_{1}, \Lambda_{2}\right) \quad \iint_{K_{\varrho} \times(-\theta, t)}|\nabla u, \nabla v, \nabla w|^{\varepsilon} \chi\left[(H-k)_{ \pm}>0\right] d x d \tau .
\end{aligned}
$$

Collecting all the above estimates, i.e. (4.6), (4.7) and (4.8)-(4.11), we obtain the following inequality:

$$
\begin{aligned}
\frac{1}{2} \int_{K_{\varrho}}(H & -k)_{ \pm}^{2} \zeta^{p}(x, t) d x+\left(\Lambda_{1} / 2\right) \iint_{K_{\varrho} \times(-\theta, t)}\left|\nabla(H-k)_{ \pm}\right|^{p} \zeta^{p} d x d \tau \\
\leq & \frac{1}{2} \int_{K_{\varrho}}(H-k)_{ \pm}^{2} \zeta^{p}(x,-\theta) d x \\
& +\frac{p}{2} \int_{-\theta}^{t} \int_{K_{\varrho}}(H-k)_{ \pm}^{2} \zeta^{p-1} \zeta_{t} d x d \tau+\widetilde{C}_{1} \iint_{K_{\varrho} \times(-\theta, t)}(H-k)_{ \pm}^{p}|\nabla \zeta|^{p} d x d \tau \\
& +\widetilde{C}_{2} \iint_{K_{\varrho} \times(-\theta, t)}|\nabla u, \nabla v, \nabla w|^{p^{2}\left(1-\kappa_{1}\right) /(n+p)} \chi\left[(H-k)_{ \pm}>0\right] d x d \tau \\
& +\widetilde{C}_{3} \iint_{K_{\varrho} \times(-\theta, t)}|F|^{p /(p-1)} \chi\left[(H-k)_{ \pm}>0\right] d x d \tau \\
& +\widetilde{C}_{4} \iint_{K_{\varrho} \times(-\theta, t)}|\nabla u, \nabla v, \nabla w|^{\varepsilon} \chi\left[(H-k)_{ \pm}>0\right] d x d \tau .
\end{aligned}
$$

Taking the supremum over $t \in(-\theta, 0)$ yields

$$
\begin{array}{r}
\sup _{-\theta<t<0} \int_{K_{\varrho}}(H-k)_{ \pm}^{2} \zeta^{p}(x, t) d x+\left(\Lambda_{1} / 2\right) \iint_{Q(\theta, \varrho)}\left|\nabla(H-k)_{ \pm}\right|^{p} \zeta^{p} d x d \tau \\
\leq \int_{K_{\varrho}}(H-k)_{ \pm}^{2} \zeta^{p}(x,-\theta) d x d \tau+C \iint_{Q(\theta, \varrho)}(H-k)_{ \pm}^{p}|\nabla \zeta|^{p} d x d \tau
\end{array}
$$




$$
\begin{aligned}
& +C \iint_{Q(\theta, \varrho)}(H-k)_{ \pm}^{2} \zeta^{p-1} \zeta_{t} d x d \tau \\
& +C \iint_{Q(\theta, \varrho)}|\nabla u, \nabla v, \nabla w|^{p^{2}\left(1-\kappa_{1}\right) /(n+p)} \chi\left[(H-k)_{ \pm}>0\right] d x d \tau \\
& +C \iint_{Q(\theta, \varrho)}|F|^{p /(p-1)} \chi\left[(H-k)_{ \pm}>0\right] d x d \tau \\
& +C \iint_{Q(\theta, \varrho)}|\nabla u, \nabla v, \nabla w|^{\varepsilon} \chi\left[(H-k)_{ \pm}>0\right] d x d \tau .
\end{aligned}
$$

Estimating the last three terms on the right by Hölder's inequality and taking into account hypotheses $(2.7),(2.13)$, and Theorem 3.1 we arrive at

$$
\begin{aligned}
& \iint_{Q(\theta, \varrho)}|\nabla u, \nabla v, \nabla w|^{p^{2}\left(1-\kappa_{1}\right) /(n+p)} \chi\left[(H-k)_{ \pm}>0\right] d x d \tau \\
& +\iint_{Q(\theta, \varrho)}|F|^{p /(p-1)} \chi\left[(H-k)_{ \pm}>0\right] d x d \tau \\
& +\iint_{Q(\theta, \varrho)}|\nabla u, \nabla v, \nabla w|^{\varepsilon} \chi\left[(H-k)_{ \pm}>0\right] d x d \tau \\
& \leq\|\nabla u, \nabla v, \nabla w\|_{\widehat{q} p^{2}\left(1-\kappa_{1}\right) /(n+p), Q}^{p^{2}\left(1-\kappa_{1}\right) /(n+p)}\left\{\int_{-\theta}^{0}\left|A_{k, \varrho}^{ \pm}(\tau)\right| d \tau\right\}^{(\widehat{q}-1) / \widehat{q}} \\
& +\|\nabla u, \nabla v, \nabla w\|_{\widehat{q} \varepsilon, Q}^{\varepsilon}\left\{\int_{-\theta}^{0}\left|A_{k, \varrho}^{ \pm}(\tau)\right| d \tau\right\}^{(\widehat{q}-1) / \widehat{q}} \\
& +\|F\|_{\widehat{q} p /(p-1), Q}^{p /(p-1)}\left\{\int_{-\theta}^{0}\left|A_{k, \varrho}^{ \pm}(\tau)\right| d \tau\right\}^{(\widehat{q}-1) / \widehat{q}} .
\end{aligned}
$$

Applying the estimate

$$
\begin{aligned}
\iint_{Q(\theta, \varrho)} \mid \nabla(H-k)_{ \pm} & \left.\right|^{p} \zeta^{p} d x d \tau \\
& \leq \iint_{Q(\theta, \varrho)}\left|\nabla(H-k)_{ \pm} \zeta\right|^{p} d x d \tau+\iint_{Q(\theta, \varrho)}(H-k)_{ \pm}^{p}|\nabla \zeta|^{p} d x d \tau
\end{aligned}
$$

to the second integral on the left of (4.12) we finally obtain (4.4).

THEOREM 4.2 (Local logarithmic estimates). Let $(u, v, w)$ be a bounded solution to the system and $H=H_{j}$ be as in (1.1). There exist constants $C$ and $\delta_{0}$ that can be determined a priori only in terms of the data such that for 
every cylinder $\left[\left(x_{0}, t_{0}\right)+Q(\theta, \varrho)\right] \subset Q$ and for every level $k$ satisfying $(4.2)$ we have the estimate

$$
\begin{aligned}
& \sup _{t_{0}-\theta<t<t_{0}} \int_{\left[x_{0}+K_{\varrho}\right]} \Psi^{2}\left(M_{k}^{ \pm},(H-k)_{ \pm}, c\right)(x, t) \zeta^{p}(x) d x \\
& \leq \int_{\left[x_{0}+K_{\varrho}\right]} \Psi^{2}\left(M_{k}^{ \pm},(H-k)_{ \pm}, c\right)\left(x, t_{0}-\theta\right) \zeta^{p}(x) d x \\
& \quad+C \iiint_{\left[\left(x_{0}, t_{0}\right)+Q(\theta, \varrho)\right]} \Psi\left|\Psi_{H}\left(M_{k}^{ \pm},(H-k)_{ \pm}, c\right)\right|^{2-p}|\nabla \zeta|^{p} d x d \tau \\
& \quad+\left(C / c^{2}\right)\left(1+\ln \frac{M_{k}^{ \pm}}{c}\right)\left\{\int_{t_{0}-\theta}^{t_{0}}\left|A_{k, \varrho}^{ \pm}(\tau)\right| d \tau\right\}^{p(1+\kappa) / q},
\end{aligned}
$$

where

$$
\begin{aligned}
\Psi\left(M_{k}^{ \pm},(H-k)_{ \pm}, c\right) & \equiv \ln ^{+}\left\{\frac{M_{k}^{ \pm}}{M_{k}^{ \pm}-(H-k)_{ \pm}+c}\right\}, \quad 0<c<M_{k}^{ \pm}, \\
\ln ^{+} s & \equiv \max \{\ln s, 0\} \quad \text { for } s>0,
\end{aligned}
$$

and additionally it is assumed that $\zeta$ is independent of $t$.

Proof. It suffices to prove (4.14) for the cube $Q(\theta, \varrho)$ since we may assume $\left(x_{0}, t_{0}\right)=(0,0)$. Choose as test functions

$$
\varphi=\left[\Psi^{2}\left(H_{h}\right)\right]^{\prime} \zeta^{p}
$$

where prime denotes differentiation with respect to $H$. By direct calculation it is easy to verify that $\left[\Psi^{2}\left(H_{h}\right)\right]^{\prime \prime}=2(1+\Psi) \Psi^{\prime 2}$ and $\varphi$ is admissible. As before, multiply the first equation of system (2.1) by $\alpha\left(\alpha=\alpha_{1}, \alpha_{2}, \alpha_{3}\right)$, the second by $\beta\left(\beta=\beta_{1}, \beta_{2}, \beta_{3}\right)$, and then add all three together. After integrating in $\tau$ over $(-\theta, t)$ with $t \in(-\theta, 0)$ and with test function $\varphi$, letting $h$ tend to zero, and making use of hypotheses $(2.5 \mathrm{a})-(2.5 \mathrm{c})$ for the leading terms we get

$$
\iint_{K_{\varrho} \times(-\theta, t)}\left\langle\alpha A^{(1)}+\beta A^{(2)}+A^{(3)}, 2(1+\Psi) \Psi^{\prime 2} \nabla H \zeta^{p}+2 p \Psi \Psi^{\prime} \zeta^{p-1} \nabla \zeta\right\rangle d x d \tau
$$

$$
\geq 2 \Lambda_{1} \iint_{K_{\varrho} \times(-\theta, t)}(1+\Psi) \Psi^{\prime 2}|\nabla H|^{p} \zeta^{p}
$$

$$
-2 \Lambda_{2} \iint_{K_{\varrho} \times(-\theta, t)}(1+\Psi) \Psi^{\prime 2}|\nabla u, \nabla v, \nabla w|^{p(p-1)\left(1-\kappa_{1}\right) /(n+p)}|\nabla H| \zeta^{p} d x d \tau
$$


$-2 \iint_{K_{\varrho} \times(-\theta, t)}(1+\Psi) \Psi^{\prime 2} F|\nabla H| \zeta^{p} d x d \tau$

$-2 p \Lambda_{2} \iint_{K_{\varrho} \times(-\theta, t)} \Psi \Psi^{\prime}|\nabla H|^{p-1}(H-k)_{ \pm} \zeta^{p-1}|\nabla \zeta| d x d \tau$

$-2 p \Lambda_{2} \iint_{K_{\varrho} \times(-\theta, t)} \Psi \Psi^{\prime}|\nabla u, \nabla v, \nabla w|^{p(p-1)\left(1-\kappa_{1}\right) /(n+p)}(H-k)_{ \pm} \zeta^{p-1}|\nabla \zeta| d x d \tau$

$-2 p \iint_{K_{\varrho} \times(-\theta, t)} \Psi \Psi^{\prime} F(H-k)_{ \pm} \zeta^{p-1}|\nabla \zeta| d x d \tau$

Let us estimate each term of the last expression by Young's inequality. For the second and third terms on the right of (4.17), we get

$$
\begin{aligned}
& 2 \Lambda_{2} \iint_{K_{Q} \times(-\theta, t)}(1+\Psi) \Psi^{\prime 2}|\nabla u, \nabla v, \nabla w|^{p(p-1)\left(1-\kappa_{1}\right) /(n+p)}|\nabla H| \zeta^{p} d x d \tau \\
& \leq\left(\Lambda_{1} / 3\right) \iint_{K_{Q} \times(-\theta, t)}(1+\Psi) \Psi^{\prime 2}|\nabla H|^{p} \zeta^{p} d x d \tau \\
& +C_{1}\left(p, \Lambda_{1}, \Lambda_{2}\right) \\
& \times \quad \iint_{K_{\varrho} \times(-\theta, t)}(1+\Psi) \Psi^{\prime 2}|\nabla u, \nabla v, \nabla w|^{p^{2}\left(1-\kappa_{1}\right) /(n+p)} \zeta^{p} d x d \tau \\
& \text { (4.19) } \quad 2 \iint_{K_{\varrho} \times(-\theta, t)}(1+\Psi) \Psi^{\prime 2} F|\nabla H| \zeta^{p} d x d \tau \\
& \leq\left(\Lambda_{1} / 3\right) \iint_{K_{\varrho} \times(-\theta, t)}(1+\Psi) \Psi^{\prime 2}|\nabla H|^{p} \zeta^{p} d x d \tau \\
& +C_{2}\left(p, \Lambda_{1}, \Lambda_{2}\right) \iint_{K_{\varrho} \times(-\theta, t)}(1+\Psi) \Psi^{\prime 2} F^{p /(p-1)} \zeta^{p} d x d \tau .
\end{aligned}
$$

For the last three terms on the right of (4.17) the following estimates are valid:

(4.20) $\quad 2 p \Lambda_{2} \iint_{K_{Q} \times(-\theta, t)} \Psi \Psi^{\prime}|\nabla H|^{p-1} \zeta^{p-1}|\nabla \zeta| d x d \tau$

$$
\begin{aligned}
\leq & C_{3}\left(p, \Lambda_{1}, \Lambda_{2}\right) \quad \iint_{K_{\varrho} \times(-\theta, t)} \Psi\left(\Psi^{\prime}\right)^{2-p}|\nabla \zeta|^{p} d x d \tau \\
& +\left(\Lambda_{1} / 3\right) \quad \iint_{K_{\varrho} \times(-\theta, t)}(1+\Psi) \Psi^{\prime 2}|\nabla H|^{p} \zeta^{p} d x d \tau ;
\end{aligned}
$$


(4.21) $2 p \iint_{K_{\varrho} \times(-\theta, t)} \Psi \Psi^{\prime}|\nabla u, \nabla v, \nabla w|^{p(p-1)\left(1-\kappa_{1}\right) /(n+p)} \zeta^{p-1}|\nabla \zeta| d x d \tau$

$$
\begin{aligned}
& \leq 2 \iint_{K_{\varrho} \times(-\theta, t)} \Psi\left(\Psi^{\prime}\right)^{2-p}|\nabla \zeta|^{p} d x d \tau \\
& \quad+2(p-1) \quad \iint_{K_{\varrho} \times(-\theta, t)} \Psi\left(\Psi^{\prime}\right)^{2}|\nabla u, \nabla v, \nabla w|^{p^{2}\left(1-\kappa_{1}\right) /(n+p)} \zeta^{p} d x d \tau ;
\end{aligned}
$$

(4.22) $2 p \quad \iint_{K_{\varrho} \times(-\theta, t)} \Psi \Psi^{\prime} F \zeta^{p-1}|\nabla \zeta| d x d \tau$

$$
\begin{aligned}
& \leq 2 \iint_{K_{\varrho} \times(-\theta, t)} \Psi\left(\Psi^{\prime}\right)^{2-p}|\nabla \zeta|^{p} d x d \tau \\
& \quad+2(p-1) \quad \iint_{K_{\varrho} \times(-\theta, t)} \Psi\left(\Psi^{\prime}\right)^{2} F^{p /(p-1)} \zeta^{p} d x d \tau .
\end{aligned}
$$

Now we turn our attention to the right-hand sides of the system, the terms containing $B$ 's. Making use of the growth assumptions (2.13) yields the following terms in the integral inequality:

$$
\begin{aligned}
& 2 \iint_{K_{\varrho} \times(-\theta, t)}\left(\alpha B^{(1)}+\beta B^{(2)}+B^{(3)}\right) \Psi \Psi^{\prime} \zeta^{p} d x d \tau \\
& \quad \leq 2 \Lambda_{2}(|\alpha|+|\beta|+1) \quad \iint_{K_{\varrho} \times(-\theta, t)}|\nabla u, \nabla v, \nabla w|^{\varepsilon} \Psi \Psi^{\prime} \zeta^{p} d x d \tau .
\end{aligned}
$$

From (4.2) and the restriction upon the set of levels $k$, taking into account the definition of $\Psi$ gives

$$
\begin{aligned}
\Psi^{\prime-1} & =M_{k}^{ \pm}-(H-k)_{ \pm}+c<2 \delta ; \\
\Psi & \leq \ln \left(M_{k}^{ \pm} / c\right), \quad \Psi^{\prime} \leq 1 / c .
\end{aligned}
$$

By (4.24) and (4.25), (4.23) can be rewritten as

$$
\begin{aligned}
2 \iint_{K_{\varrho} \times(-\theta, t)}\left(\alpha B^{(1)}\right. & \left.+\beta B^{(2)}+B^{(3)}\right) \Psi \Psi^{\prime} \zeta^{p} d x d \tau \\
\leq & C_{4}\left(c,|\alpha|,|\beta|, \Lambda_{2}\right)\left(1+\ln \left(M_{k}^{ \pm} / c\right)\right) \\
& \times \quad \iint_{K_{\varrho} \times(-\theta, t)}|\nabla u, \nabla v, \nabla w|^{\varepsilon} \chi\left[(H-k)_{ \pm}>0\right] d x d \tau .
\end{aligned}
$$

Making use of (4.24) and (4.25) in (4.18), (4.19), and in (4.21) and (4.22), implies the following estimates: 
$(4.27)$

$$
\begin{aligned}
& \quad \iint_{K_{\varrho} \times(-\theta, t)}(1+\Psi) \Psi^{\prime 2}|\nabla u, \nabla v, \nabla w|^{p^{2}\left(1-\kappa_{1}\right) /(n+p)} \zeta^{p} d x d \tau \\
& \leq\left(1 / c^{2}\right)\left(1+\ln \left(M_{k}^{ \pm} / c\right)\right) \\
& \quad \times \quad \iint_{K_{\varrho} \times(-\theta, t)}|\nabla u, \nabla v, \nabla w|^{p^{2}\left(1-\kappa_{1}\right) /(n+p)} \chi\left[(H-k)_{ \pm}>0\right] d x d \tau ;
\end{aligned}
$$

$$
\begin{aligned}
& \quad \iint_{K_{\varrho} \times(-\theta, t)} \Psi \Psi^{\prime 2}|F|^{p /(p-1)} \zeta^{p} d x d \tau \leq \iiint_{K_{\varrho} \times(-\theta, t)}(1+\Psi) \Psi^{\prime 2} F^{p /(p-1)} \zeta^{p} d x d \tau \\
& \leq\left(1 / c^{2}\right)\left(1+\ln \left(M_{k}^{ \pm} / c\right)\right) \quad \iint_{K_{\varrho} \times(-\theta, t)}|F|^{p /(p-1)} \chi\left[(H-k)_{ \pm}>0\right] d x d \tau .
\end{aligned}
$$

Apply inequalities (4.27) and (4.28) to the appropriate terms in estimates (4.18), (4.19), and in (4.21) and (4.22). Combining (4.18), (4.19), (4.20)(4.22), and (4.26) yields

$$
\begin{aligned}
\int_{K_{\varrho} \times\{t\}} \Psi^{2} \zeta^{p} d x \leq & \int_{K_{\varrho} \times\{-\theta\}} \Psi^{2} \zeta^{p} d x+C \iiint_{K_{Q} \times(-\theta, t)} \Psi\left|\Psi^{\prime}\right|^{2-p}|\nabla \zeta|^{p} d x d \tau \\
& +\left(C / c^{2}\right)\left(1+\ln \left(M_{k}^{ \pm} / c\right)\right) \\
& \times\left\{\iiint_{K_{\varrho} \times(-\theta, t)}|\nabla u, \nabla v, \nabla w|^{p^{2}\left(1-\kappa_{1}\right) /(n+p)} \chi\left[(H-k)_{ \pm}>0\right] d x d \tau\right. \\
& +\iint_{K_{\varrho} \times(-\theta, t)}|F|^{p /(p-1)} \chi\left[(H-k)_{ \pm}>0\right] d x d \tau \\
& \left.+\iiint_{K_{\varrho} \times(-\theta, t)}|\nabla u, \nabla v, \nabla w|^{\varepsilon} \chi\left[(H-k)_{ \pm}>0\right] d x d \tau\right\} .
\end{aligned}
$$

Taking the supremum over $t \in(-\theta, 0)$ implies

$$
\begin{aligned}
& \sup _{-\theta<t<0} \int_{K_{\varrho} \times\{t\}} \Psi^{2} \zeta^{p} d x \\
& \leq \int_{K_{\varrho} \times\{-\theta\}} \Psi^{2} \zeta^{p} d x+\widetilde{C} \iint_{Q(\theta, \varrho)} \Psi\left|\Psi^{\prime}\right|^{2-p}|\nabla \zeta|^{p} d x d \tau \\
& \quad+\left(\widetilde{C} / c^{2}\right)\left(1+\ln \left(M_{k}^{ \pm} / c\right)\right) \\
& \quad \times\left\{\iint_{Q(\theta, \varrho)}|\nabla u, \nabla v, \nabla w|^{p^{2}\left(1-\kappa_{1}\right) /(n+p)} \chi\left[(H-k)_{ \pm}>0\right] d x d \tau\right. \\
& \quad+\iint_{Q(\theta, \varrho)}|F|^{p /(p-1)} \chi\left[(H-k)_{ \pm}>0\right] d x d \tau \\
& \left.\quad+\iint_{Q(\theta, \varrho)}|\nabla u, \nabla v, \nabla w|^{\varepsilon} \chi\left[(H-k)_{ \pm}>0\right] d x d \tau\right\}
\end{aligned}
$$


Estimating the last four terms on the right of (4.29) by Hölder's inequality, as in (4.13), we arrive at (4.14).

From Theorems 4.1 and 4.2, with the help of Lemma 2.2, Proposition 2.1, Lemma 4.1, and Lemma 4.2 of Chapter I of [5], it follows that a weak solution to system (2.1) is Hölder continuous in the interior of the domain $Q$ :

TheOREM 4.3 (Interior Hölder continuity). If $H \in C\left(0, T ; L^{2}(\Omega)\right) \cap$ $L^{p}\left(0, T ; W^{1, p}(\Omega)\right)$ is bounded and satisfies inequalities (4.4) of Theorem 4.1 and (4.14) of Theorem 4.2 then there exist constants $C$ and $\alpha \in(0,1)$ depending only upon the data, such that for all subdomains $Q^{\prime} \subset Q$, for every pair of points $\left(x_{1}, t_{1}\right),\left(x_{2}, t_{2}\right) \in Q^{\prime}$,

$$
\left|H\left(x_{1}, t_{1}\right)-H\left(x_{2}, t_{2}\right)\right| \leq C\left(\frac{\left|x_{1}-x_{2}\right|+\left|t_{1}-t_{2}\right|^{1 / p}}{\operatorname{dist}\left(Q^{\prime}, \partial Q, p\right)}\right)^{\alpha}
$$

with

$$
\operatorname{dist}\left(Q^{\prime}, \partial Q, p\right) \equiv \inf _{\substack{(x, t) \in Q^{\prime} \\(y, s) \in \partial Q}}\left(|x-y|+|t-s|^{1 / p}\right) .
$$

For the proof of this theorem see [5, p. 77, Theorem 1.1].

Regularity up to the boundary. Let us introduce some additional notation. Set

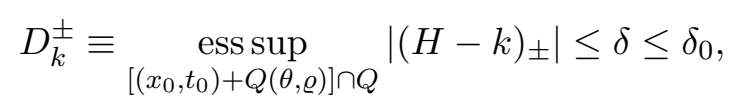

and introduce the following restrictions on the set of levels:

$$
\left\{\begin{array}{l}
k \geq \sup _{\left[\left(x_{0}, t_{0}\right)+Q(\theta, \varrho)\right] \cap S}\left[\alpha g_{1}+\beta g_{2}+g_{3}\right] \\
k \leq \sup _{\left[\left(x_{0}, t_{0}\right)+Q(\theta, \varrho)\right] \cap S}\left[\alpha g_{1}+\beta g_{2}+g_{3}\right] \\
\quad \text { for the test function }(H-k)_{+} \zeta^{p},
\end{array}\right.
$$

Here $\delta_{0}$ is the positive parameter from (4.2). Define

$$
\Psi\left(D_{k}^{ \pm},(H-k)_{ \pm}, c\right) \equiv \ln ^{+}\left\{\frac{D_{k}^{ \pm}}{D_{k}^{ \pm}-(H-k)_{ \pm}+c}\right\}, \quad c<D_{k}^{ \pm},
$$

and

$$
B_{k, \varrho}^{ \pm}(\tau) \equiv\left\{x \in\left[x_{0}+K_{\varrho}\right] \cap \Omega \mid(H(x, \tau)-k)_{ \pm}>0\right\} .
$$

THEOREM 4.4. There are constants $C$ and $\delta_{0}$ determined only by the data such that for fixed $\left(x_{0}, t_{0}\right) \in S$ for every cylinder $\left[\left(x_{0}, t_{0}\right)+Q(\theta, \varrho)\right]$ with $\theta$ 
so small that $t_{0}-\theta>0$, and for every level $k$ satisfying $(4.30 \mathrm{a})-(4.30 \mathrm{~b})$,

$$
\begin{aligned}
\sup _{t_{0}-\theta<t<t_{0}} \int_{\left[x_{0}+K_{\varrho}\right] \cap \Omega}(H-k)_{ \pm}^{2} \zeta^{p}(x, t) d x \\
+C^{-1} \iiint_{\left[\left(x_{0}, t_{0}\right)+Q(\theta, \varrho)\right] \cap Q}\left|\nabla(H-k)_{ \pm} \zeta\right|^{p} d x d \tau
\end{aligned}
$$

$\leq \int_{\left[x_{0}+K_{\varrho}\right] \cap \Omega}(H-k)_{ \pm}^{2} \zeta^{p}\left(x, t_{0}-\theta\right) d x$

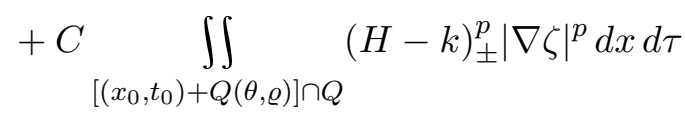

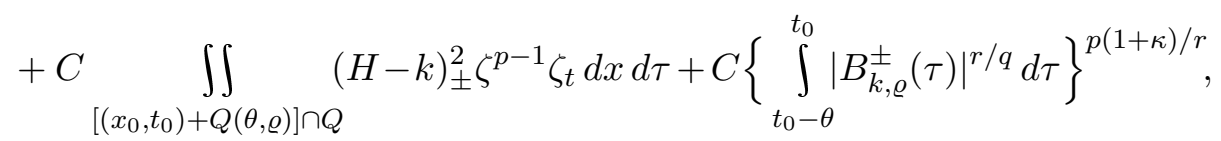

and, provided that the cut-off function is independent of $t$ for $t \in\left(t_{0}-\theta, t_{0}\right)$,

$$
\begin{aligned}
\sup _{t_{0}-\theta<t<t_{0}} \int_{\left[x_{0}+K_{\varrho}\right] \cap \Omega} \Psi^{2}\left(D_{k}^{ \pm},(H-k)_{ \pm}, c\right)(x, t) \zeta^{p}(x) d x \\
\leq \int_{\left[x_{0}+K_{\varrho}\right] \cap \Omega} \Psi^{2}\left(D_{k}^{ \pm},(H-k)_{ \pm}, c\right)\left(x, t_{0}-\theta\right) \zeta^{p}(x) d x \\
\quad+C \iiint_{\left[\left(x_{0}, t_{0}\right)+Q(\theta, \varrho)\right] \cap Q} \Psi\left|\Psi_{H}\left(D_{k}^{ \pm},(H-k)_{ \pm}, c\right)\right|^{2-p}|\nabla \zeta|^{p} d x d \tau \\
+\left(C / c^{2}\right)\left(1+\ln \frac{D_{k}^{ \pm}}{c}\right)\left\{\int_{t_{0}-\theta}^{t_{0}}\left|B_{k, \varrho}^{ \pm}(\tau)\right| d \tau\right\}^{p(1+\kappa) / q} .
\end{aligned}
$$

The proof is a literal repetition of that of Theorems 4.1 and 4.2 with the only difference that we have to consider $D_{k}^{ \pm}$instead of $M_{k}^{ \pm}$, and $B_{k, \varrho}^{ \pm}(\tau)$ instead of $A_{k, \varrho}^{ \pm}(\tau)$.

\section{Initial regularity}

THEOREM 4.5. There are constants $C$ and $\delta_{0}$ determined only by the data such that for every $\left(x_{0}, t_{0}\right) \in Q$ and for every cylinder $\left[\left(x_{0}, t_{0}\right)+Q(\theta, \varrho)\right]$ with $t_{0}-\theta \equiv 0$, if the cut-off function $\zeta$ is independent of $t$ for $t \in\left(0, t_{0}\right)$, then

$$
\sup _{0<t<t_{0}} \int_{\left[x_{0}+K_{\varrho}\right]}(H-k)_{ \pm}^{2} \zeta^{p}(x, t) d x+\int_{\left[\left(x_{0}, t_{0}\right)+Q(\theta, \varrho)\right] \cap Q}\left|\nabla(H-k)_{ \pm} \zeta\right|^{p} d x d \tau
$$

$$
\leq C \iint_{\left[\left(x_{0}, t_{0}\right)+Q(\theta, \varrho)\right] \cap Q}(H-k)_{ \pm}^{p}|\nabla \zeta|^{p} d x d \tau+C\left\{\int_{0}^{t_{0}}\left|B_{k, \varrho}^{ \pm}(\tau)\right|^{r / q} d \tau\right\}^{p(1+\kappa) / r}
$$


and moreover,

$$
\begin{aligned}
\sup _{0<t<t_{0}} & \int_{\left[x_{0}+K_{\varrho}\right] \cap \Omega} \Psi^{2}\left(D_{k}^{ \pm},(H-k)_{ \pm}, c\right)(x, t) \zeta^{p}(x) d x \\
\leq & C \iint_{\left[\left(x_{0}, t_{0}\right)+Q(\theta, \varrho)\right] \cap Q} \Psi\left|\Psi_{H}\left(D_{k}^{ \pm},(H-k)_{ \pm}, c\right)\right|^{2-p}|\nabla \zeta|^{p} d x d \tau \\
& +\left(C / c^{2}\right)\left(1+\ln \frac{D_{k}^{ \pm}}{c}\right)\left\{\int_{0}^{t_{0}}\left|B_{k, \varrho}^{ \pm}(\tau)\right| d \tau\right\}^{p(1+\kappa) / q},
\end{aligned}
$$

where $q, \kappa$ satisfy (4.1), $k$ fulfills (4.30a) and in addition the following restrictions are assumed:

$$
\begin{array}{ll}
k \geq \sup _{\left[x_{0}+K_{\varrho}\right] \cap \Omega} H_{0} & \text { for }(H-k)_{+}, \\
k \leq \sup _{\left[x_{0}+K_{\varrho}\right] \cap \Omega} H_{0} & \text { for }(H-k)_{-} .
\end{array}
$$

The proof is analogous to that of Theorems 4.1 and 4.2.

Thus, summing up, from Theorems 4.4 and 4.5 we come to the statement:

THEOREM 4.6 (Hölder continuity up to the boundary). If $H(x, t)$ from Theorem 4.3 satisfies inequalities (4.32), (4.33) from Theorem 4.4, and (4.34), (4.35) from Theorem 4.5, the boundary data are Hölder continuous on $S$ with exponent $\widetilde{\alpha}_{g}^{j}$, and the initial data are Hölder continuous in $\bar{\Omega}$ with exponent $\widetilde{\alpha}_{0}^{j}$, then there exist constants $c>0$ and $\alpha \in(0,1)$ depending only upon the data of the problem such that for any $\left(x_{1}, t_{1}\right),\left(x_{2}, t_{2}\right) \in \bar{Q}$,

$$
\left|H\left(x_{1}, t_{1}\right)-H\left(x_{2}, t_{2}\right)\right| \leq c\left(\left|x_{1}-x_{2}\right|+\left|t_{1}-t_{2}\right|^{1 / p}\right)^{\alpha} .
$$

For the proof of this theorem see [5, p. 78, Theorem 1.2]. Hence the Hölder continuity of the components of the solution themselves immediately follows:

$$
\begin{aligned}
\|u\|_{H^{\alpha, \alpha} p}= & \|u \Delta\|_{H^{\alpha, \alpha} / p} /|\Delta| \\
= & \|\left(\alpha_{1} u+\beta_{1} v+w\right)\left(\beta_{2}-\beta_{3}\right)-\left(\alpha_{2} u+\beta_{2} v+w\right)\left(\beta_{1}-\beta_{3}\right) \\
& +\left(\alpha_{3} u+\beta_{3} v+w\right)\left(\beta_{1}-\beta_{2}\right) \|_{H^{\alpha, \alpha}, p} /|\Delta| \\
= & \left\|\left(\beta_{2}-\beta_{3}\right) H_{1}-\left(\beta_{1}-\beta_{3}\right) H_{2}+\left(\beta_{1}-\beta_{2}\right) H_{3}\right\|_{H^{\alpha, \alpha} / p} /|\Delta| \\
\leq & \left(\left|\beta_{2}-\beta_{3}\right| C_{1}+\left|\beta_{1}-\beta_{3}\right| C_{2}+\left|\beta_{1}-\beta_{2}\right| C_{3}\right) /|\Delta| \\
\|v\|_{H^{\alpha, \alpha} p}= & \|v \Delta\|_{H^{\alpha, \alpha}, p} /|\Delta| \\
= & \|\left(\alpha_{1} u+\beta_{1} v+w\right)\left(\alpha_{2}-\alpha_{3}\right)-\left(\alpha_{2} u+\beta_{2} v+w\right)\left(\alpha_{1}-\alpha_{3}\right) \\
& +\left(\alpha_{3} u+\beta_{3} v+w\right)\left(\alpha_{1}-\alpha_{2}\right) \|_{H^{\alpha, \alpha / p}} /|\Delta| \\
= & \left\|\left(\alpha_{2}-\alpha_{3}\right) H_{1}-\left(\alpha_{1}-\alpha_{3}\right) H_{2}+\left(\alpha_{1}-\alpha_{2}\right) H_{3}\right\|_{H^{\alpha, \alpha} / p} /|\Delta| \\
\leq & \left(\left|\alpha_{2}-\alpha_{3}\right| C_{1}+\left|\alpha_{1}-\alpha_{3}\right| C_{2}+\left|\alpha_{1}-\alpha_{2}\right| C_{3}\right) /|\Delta| ;
\end{aligned}
$$




$$
\begin{aligned}
\|w\|_{H^{\alpha, \alpha} / p} & =\left\|\left(\alpha_{1} u+\beta_{1} v+w\right)-\alpha_{1} u-\beta_{1} v\right\|_{H^{\alpha, \alpha / p}} \\
& \leq\left\|H_{1}-\alpha_{1} u-\beta_{1} v\right\|_{H^{\alpha, \alpha} / p} \\
& \leq\left\|H_{1}\right\|_{H^{\alpha, \alpha} / p}+\left|\alpha_{1}\right|\|u\|_{H^{\alpha, \alpha} / p}+\left|\beta_{1}\right|\|v\|_{H^{\alpha, \alpha}, p}
\end{aligned}
$$

where $\|\cdot\|_{H^{\alpha, \alpha / p}}$ denotes the Hölder norm:

$$
\|u\|_{H^{\alpha, \alpha / p}}=\sup _{\left(x_{1}, t_{1}\right),\left(x_{2}, t_{2}\right) \in Q} \frac{\left|u\left(x_{1}, t_{1}\right)-u\left(x_{2}, t_{2}\right)\right|}{\left(\left|x_{1}-x_{2}\right|+\left|t_{1}-t_{2}\right|^{1 / p}\right)^{\alpha}} .
$$

\section{References}

[1] A. V. Bitsadze, On elliptic systems of differential equations with partial derivatives of second order, Dokl. Akad. Nauk SSSR 112 (1957), 983-986 (in Russian).

[2] - Boundary Value Problems for Second Order Elliptic Equations, Nauka, Moscow, 1966 (in Russian).

[3] —, Some Classes of Partial Differential Equations, Nauka, Moscow, 1981.

[4] Y. Z. Chen and L. C. Wu, Second Order Elliptic Equations and Elliptic Systems, Amer. Math. Soc. Providence, RI, 1998.

[5] E. DiBenedetto, Degenerate Parabolic Equations, Springer, New York, 1993.

[6] E. DiBenedetto and Y. Z. Chen, Boundary estimates for solutions of nonlinear degenerate parabolic systems, J. Reine Angew. Math. 395 (1989), 102-131.

[7] L. Dung, Hölder regularity for certain strongly coupled parabolic systems, J. Differential Equations 151 (1999), 313-344.

[8] A. Friedman and E. DiBenedetto, Regularity of solutions of nonlinear degenerate parabolic systems, J. Reine Angew. Math. 349 (1984), 83-128.

[9] —, - Addendum to: "Hölder estimates for nonlinear degenerate parabolic systems", ibid. 363 (1985), 217-220.

[10] - - - Hölder estimates for nonlinear degenerate parabolic systems, J. Reine Angew. Math. 357 (1985), 1-22.

[11] E. De Giorgi, Un esempio di estremali discontinue per un problema variazionale di tipo ellittico, Boll. Un. Mat. Ital. 1 (1968), 135-137.

[12] K. H. W. Küfner, Global existence for a certain strongly coupled quasilinear parabolic system in population dynamics, Analysis 15 (1995), 343-357.

[13] O. A. Ladyzhenskaya, N. A. Solonnikov, and N. N. Ural'tseva, Linear and Quasilinear Equations of Parabolic Type, Amer. Math. Soc., Providence, RI, 1968.

[14] M. A. Pozio and A. Tesei, Global existence of solutions for a strongly coupled quasilinear parabolic system, Nonlinear Anal. 12 (1990), 657-689.

[15] M. Wiegner, Global solutions to a class of strongly coupled parabolic systems, Math. Ann. 292 (1992), 711-727.

[16] W. Zajączkowski, Global existence of solutions for Dirichlet problem to nonlinear diagonal parabolic system with maximal growth conditions, J. Appl. Anal. 1 (1995), 159-172.

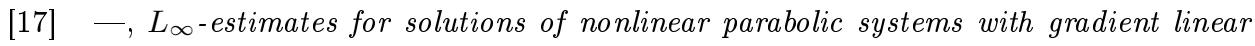
growth, in: Banach Center Publ. 33, Inst. Math., Polish Acad. Sci., 1996, 491-501.

[18] -, $L_{\infty}$-estimate for qualitatively bounded weak solutions of nonlinear degenerate diagonal parabolic systems, J. Appl. Anal. 2 (1996), 1-12.

[19] - , L $L_{\infty}$-estimate for solutions of nonlinear parabolic systems, in: Banach Center Publ. 33, Inst. Math., Polish Acad. Sci., 1996, 465-490. 
[20] W. Zajączkowski and D. Wrzosek, Existence of solutions and $L^{\infty}$-bounds for quasilinear degenerate parabolic systems, J. Appl. Anal. 5 (1999), 197-221.

Institute for Condensed Matter Physics of the National Academy of Sciences of Ukraine

1 Svientsitskii Street, 79011 Lviv, Ukraine

E-mail: port@icmp.lviv.ua

Received 4.5.2005

and in final form 17.9.2005 